\title{
Devista Eletrônica
}

REF - ISSN 1808-0804 Vol. IX (1), 1 - 17, 2012.

\section{INTERAÇÕES MEDICAMENTOSAS E EFEITOS ADVERSOS QUE OCORREM EM PRESCRIÇÕES DO SUS COM O MEDICAMENTO FLUOXETINA NO DISTRITO DE SÃO PAULO, ZONA NORTE.}

DRUG INTERACTIONS AND SIDE EFFECTS OF FLUOXETINE IN SUS PRESCRIPTIONS, DISTRICT OF SÃO PAULO.

INTERACTIONES MEDICAMENTOSAS Y EFECTOS ADVERSOS QUE OCURREM EN LAS RECETAS MEDICAS DE FLUOXETINA, EN EL SUS, EL NORTE, CIUDAD DE SÃO PAULO.

\section{Fabiana Gatti de Menezes ${ }^{1 *}$, Luciano Carlos Vieira de Mariz².}

${ }^{1}$ Pós-doutoranda em Farmácia Hospitalar, HU-USP; Docente dos cursos de pósgraduação em Farmácia Hospitalar e Farmácia Hospitalar Oncológica, das Faculdades Oswaldo Cruz e Hospital A. C. Camargo; Docente do Curso de Farmácia da Universidade Nove de Julho; Avenida Dr. Adolfo Pinto, 109 - Barra Funda, CEP 01156-050, São Paulo SP, Brasil.

${ }^{2}$ Graduado em Farmácia e Bioquímica pela Universidade Nove de Julho; Gerente Farmacêutico; Drogaria São Paulo; Avenida Sargento Geraldo Santana, 1100 BL 4 AP 11,Jardim Taquaral, CEP 04674-000, SP-SP.

* Autor para correspondência: fgatti@usp.br

\section{Recebido em 15/02/2012, Aceito em 13/03/2012.}

RESUMO: O presente trabalho foi realizado na UBS parque Novo Mundo II, Zona Norte de São Paulo. Nele foi descrito o perfil do paciente que utiliza fluoxetina e identificado possíveis interações medicamentosas e efeitos adversos. Foi utilizado um questionário estruturado, 
De Menezes, F.G. De Mariz, L.C.V. Revista Eletrônica de Farmácia Vol. IX (1), 1 - 17, 2012.

questões abertas e fechadas. Forma entrevistados 44 pacientes, sendo $84.40 \%$ mulheres, com grande maioria apresentando baixo nível de escolaridade e idade entre 40 à 55 anos de idade. Dos pacientes, $75 \%$ apresentaram efeitos adversos, os mais pronunciados forma secura de boca, ansiedade, nervosismo, palpitação, perda e peso, sonolência, e sudorese. Também foram observadas interações medicamentosas metabólicas.

PALAVRAS - CHAVE: Fluoxetina, Interações Medicamentosas, Efeitos Adversos.

ABSTRACT: This study was conducted at UBS New World Park II, Zona Norte de São Paulo. In it was using fluoxetine and identify possible drug interactions and side effects. Used a structured questionnaire for questions opens and close. Interviewed 44 patients, $84.40 \%$ womwn, with most showing low education and age between 40 to 55 years age. Patients, $75 \%$ had side effects, the most pronounced form a dry mouth, anxiety, nervousness, palpitations, and weight loss, drowsiness, and sudorese. Metabolic drug interactions were observed.

KEYWORDS: Fluoxetine, Drug Interactions, Side Effects.

RESUMEN: Este estudio se llevó a cabo en UBS New World Park II, la zona norte de São Paulo. En ella se describe un perfil de los pacientes que usan la droga y se identificaron posibles interacciones medicamentosas y los efectos adversos. Se utilizó una serie de preguntas estructuradas, abiertas y cerradas. Forma entrevistó a 44 pacientes, mujeres $84.40 \%$, con la mayoría que muestra el bajo nivel de educación y edad entre 40 a 55 años los pacientes idade. Dos, el 75\% tuvo efectos adversos, más pronunciada a medida que la boca seca, ansiedad, nerviosismo, la pérdida de palpitaciones, y el peso, somnolencia y sudoración. También las interacciones metabólicas con otros fármacos se observaron.

DESCRITORES: Fluoxetina, Interacciones, Efectos Secundarios.

\section{INTRODUÇÃO}

A Unidade básica de saúde (UBS) funciona como uma porta de entrada para o SUS, executa o atendimento e quando necessário o posterior encaminhamento para especialistas e outros serviços ${ }^{(1)}$. 0 atendimento e acolhimento primordiais e de baixa gravidade ou de pequena complexidade também são atribuições das UBS $^{(1)}$. Dentro das UBS também se encontram as farmácias que dispensam medicamentos padronizados pelo SUS diretamente para a população ${ }^{(1)}$

Hoje a fluoxetina faz parte da lista de medicamentos padronizados pelo SUS, seguindo os critérios preconizados pela Organização Mundial da Saúde (OMS), de 
De Menezes, F.G. De Mariz, L.C.V. Revista Eletrônica de Farmácia Vol. IX (1), 1 - 17, 2012.

atender à maioria dos problemas de saúde da população brasileira, assim é distribuído gratuitamente pelo governo(2). O que aumentou o acesso da grande população a esse remédio(2).

No final da década de 80 o medicamento fluoxetina foi introduzido no mercado, provocando grandes alterações no que diz respeito ao tratamento da depressão ${ }^{(3)}$. Esse medicamento funciona com eficácia no tratamento das seguintes doenças: doença compulsivo-obsessiva, bulimia nervosa, doença disfórica prémenstrual, doença do pânico, distimia, ansiedade, manifestações clínicas da depressão. Essa droga também é indicação para o tratamento da anorexia nervosa (3). A fluoxetina pertence a um grupo terapêutico que apresenta uma maior segurança terapêutica, quando comparado aos antigos antidepressivos tricíclicos $(A D T)^{(4)}$, com menor índice de efeitos adversos e redução do número de interações medicamentosas, porém esta redução não torna nula essas interações e os possíveis efeitos adversos ${ }^{(4)}$.

A fluoxetina é um antidepressivo que atua como um inibidor seletivo da recaptação de serotonina (ISRS), nos dias atuais é um dos principais tratamentos de escolha para a depressão e para as perturbações da ansiedade, em muitos casos com associações medicamentosas. A companhia farmacêutica Eli Lilly é responsável pela síntese inicial da fluoxetina. Foi lançada no comérecio com o nome de Prozac $\AA^{(5)}$.

$$
\text { No ano de } 1.970 \text { a Eli Lilly foi }
$$
responsável pela descoberta, sintese inicial e comercialização da fluoxetina, a empresa teve a colaboração do pesquisadores Bryan Molloy e Robert Rathburn $^{(4)}$. Era sabido na época que o fármaco difenidramina (anti-histamínico) posuia alguns efeitos antidepressivos ${ }^{(4)}$. Usando como ponto de partida esse antihistamínico foi sintetizado o composto 3fenoxi-3-fenilpropilamina, com estrutura similar à difenidramina. Molloy sintetizou vários derivados, e os testes pré-clínicos resultaram na descoberta da nisoxetina, que atua como um inibidor seletivo da recaptação da noradrenalina, hoje em dia é amplamente utilizado em experiências bioquímicas $^{(4)}$. Posteriormente, na busca de um derivado que fosse apenas um inibidor da recaptação de serotonina, Wong propôs que novos testes in vitro fossem testados para as seguintes substâncias: serotonina, noradrenalina e dopamina. Estes testes foram realizados por Jong-Sir Horng em maio de 1972, mostraram que um composto denominado fluoxetina, era o mais potente inibidor da serotonina da série. A fluoxetina teve seu lançamento no mercado Belga em $1986^{(5)}$ e foi aprovada pela FDA em dezembro de 1987 nos Estados Unidos. A fluoxetina foi o quarto ISRS a surgir no mercado, após a indalpina, zimelidina e fluvoxamina. " $A$ 
De Menezes, F.G. De Mariz, L.C.V. Revista Eletrônica de Farmácia Vol. IX (1), 1 - 17, 2012.

patente da Eli Lilly sob o Prozac $®$ expirou em Agosto de 2001", aumentando o influxo de genéricos ao mercado. Somente nos Estados Unidos, foi verificado que mais de 21,7 milhões de prescrições genéricas do medicamento fluoxetina foram efetuadas no ano de 2006, colocando a substância em terceira posição dentre os antidepressivos mais receitados no mundo, ficando atrás apenas, da sertralina e do escitalopran ${ }^{(6)}$.

Porém, a maioria das bulas $e$ dicionários de especialidades não possui dados suficientes sobre as possíveis interações medicamentosas e seus riscos, acarretando resposta farmacologicamente diferente da conhecida(3).

\section{Farmacologia da fluoxetina}

Pelo seu mecanismo de ação a fluoxetina potencializa seletivamente a atividade da serotonina [5hidroxitriptamina (5-HT)], funciona através da inibição da bomba de recaptação, aumentando assim os níveis de 5-HT disponível na fenda sinaptica para atuar na neurotransmissão de 5-HT. Além de atuar na ISRS, também inibe a recaptação de noradrenalina. A serotonina é um neurotransmissor, quimicamente pode ser chamado de 5-hidroxitriptamina (5-HT) que também é uma indolamina, "produto da transformação do aminoácido L-Triptofano"(7). Existem vários receptores 5-HT, destacando-se os receptores 5HT1A， 5-HT2A， 5-HT2C e 5-HT3. A estimulação dos diferentes receptores leva à efeitos diferentes desse antidepressivo $^{(8)}$, vide tabela 1.

Tabela 1: Receptores da serotonina e seu seus efeitos farmacológicos

\begin{tabular}{|c|c|}
\hline Receptor & Efeito \\
\hline 5-HT1A & Efeitos ansiolíticos e antidepressivos ${ }^{(7,9)}$. \\
\hline 5-HT2A & $\begin{array}{l}\text { Efeitos antidepressivos, antiobsessivos e de controle do } \\
\text { comportamento alimentar compulsivo, efeitos ansiogênicos em } \\
\text { em longo prazo, redução da libido e alterações no funcionamento } \\
\text { sexual. Antipsicóticos atípicos aumentam os níveis de dopamina } \\
\text { em determinadas áreas através do bloqueio destes receptores }\end{array}$ \\
\hline
\end{tabular}


De Menezes, F.G. De Mariz, L.C.V. Revista Eletrônica de Farmácia Vol. IX (1), 1 - 17, 2012.

\begin{tabular}{|c|c|}
\hline 5-HT3C & $\begin{array}{c}\text { Efeitos ansiogênicos, regulação do peso, regulação do } \\
\text { sistema dopaminérgico }\end{array}$ \\
\hline 5-HT4C & Efeitos secundários gastrintestinais: náuseas e vômitos ${ }^{(7,9)}$. \\
\hline
\end{tabular}

Tabela adaptada de: Mancini MC, Halpern A. Aspectos fisiológicos do balanço energético. Arq Bras Endocrinol e Carliniu, E A. Fluoxetina: indícios de uso inadequado. J. bras. psiquiatr..

O efeito deste antidepressivo ocorre através de um aumento dos níveis de serotonina disponíveis para atuar no nível dos auto-receptores 5-HT1A. O efeito tardio corresponde a uma dessensibilização destes receptores, com conseqüente desinibição dos mesmos e aumento da liberação de serotonina para a fenda sináptica. Ficando assim a serotonina disponível para atuar nos receptores pós-sinápticos 5-HT. Inicialmente ela ativa estes receptores, que posteriormente podem dessensibilizar$\mathrm{se}^{(7)}$.

Alguns autores afirmam que esta dessensibilização corre na origem do desaparecimento dos efeitos secundários. Assim sendo os efeitos terapêuticos devem ocorrem por uma atividade serotonérgica prolongada e aumentada em diferentes subtipos de receptores daqueles que medeiam os efeitos secundários ${ }^{(7)}$.

Entre os efeitos farmacológicos da fluoxetina encontramos os efeitos adversos. Define-se efeito adverso, ou reação adversa ao medicamento (RAM), segundo definição da Anvisa: "É qualquer resposta a um medicamento que seja prejudicial, não intencional, e que ocorra nas doses normalmente utilizadas em seres humanos para profilaxia, diagnóstico e tratamento de doenças, ou para a modificação de uma função fisiológica" (10). Os efeitos adversos da fluoxetina são: Alopecia, alteração de concentração ou raciocínio, alteração do paladar, ansiedade, ausência ou atraso na ejaculação, calafrio, desequilíbrio, dificuldade de deglutição, dificuldade de digestão, dificuldade ou dor para urinar, diminuição do desejo sexual, ereção persistente do pênis acompanhada de dor, ereção prolongada, erupções na pele, fadiga, falta de orgasmo, falta ou perda de apetite, impotência, náusea, nervosismo, palpitação, perda de peso, reação maníaca, secura de boca, sonhos anormais, sonolência, sudorese, tremor ou movimentos anormais, vertigem, vômito ${ }^{(11)}$.

\section{Farmacoterapia da fluoxetina}

O ISRS deve ser iniciado em doses baixas (ex. $5 \mathrm{mg}$ a $10 \mathrm{mg}$ de fluoxetina), o aumento da dose deve ser lento e progressivo. Quando for alcançada a dose ideal, deve-se aguardar de quatro a seis 
De Menezes, F.G. De Mariz, L.C.V. Revista Eletrônica de Farmácia Vol. IX (1), 1 - 17, 2012.

semanas para avaliar a resposta terapêutica. A retirada deve ser gradual, a cada cinco/sete dias ${ }^{(7,9)}$.

A fluoxetina é metabolizada por algumas isoenzimas do sistema CYP450, esse sistema participa da fração microssomal hepática e promove reações de oxidação de fármacos, desempenhando parte do processo farmacocinético denominado biotransformação(12). Seu metabolismo ocorre através das isoenzimas CYP2C9, CYP2D6, CYP3A4, é inibidora enzimática das isoenzimas CYP2C9, CYP2C18， CYP2C19， CYP2D6,
CYP3A4. A tabela II. Já na sua biotransformação os níveis de fluoxetina já podem ser diminuídos ou aumentados. Se o fármaco for administrado com fármacos que inibam seu citocromo biotransformador os níveis de fluoxetina irão aumentar no organismo. Se for administrada com fármacos que forem indutores enzimáticos a permanência do fármaco tende a ser maior no organismo. Já a fluoxetina sendo inibidora enzimática pode aumentar o tempo de permanência de outros fármacos no organismo ${ }^{(13,14,15)}$.

Tabela 2: Isoenzima CYP2C9 do Citocromo P-450 que é estimulada pela Fluoxetina(14).

\begin{tabular}{|l|c|c|c|c|}
\hline Isoenzima & \multicolumn{2}{|c|}{ Substrato } & \multicolumn{2}{c|}{ Medicamentos inibidores } \\
\hline CYP2C9 & Ácido & Fluoxetina & Amiodarona & Metronidazol \\
& mefenâmico & ibuprofeno & Cetoconazol & Miconazol \\
& ADT & Imipramina & Cimetidina & Omeprazol \\
& AINE & Losartano & Diazepam & Sertralina \\
& Amitriptilina & Meloxicam & Diclofenaco & Sildenafil \\
& Barbituratos & Naproxeno & Fluconazol & Sulfametoxazo \\
& Bupropiona & Omeprazol & Fluoxetina & I-trimetropina \\
& Carvedilol & Piroxicam & Imipramina & Topiramato \\
& Celecoxibe & Propranolol & Isoniazida & Varfarina \\
& Citalopram & Sertralina & & \\
& Diazepam & Sildenafil & & \\
& Diclofenaco & Sulfonilurea & & \\
& Escitalopram & Varfarina & & \\
& Fenitoína & & & \\
& Fenobarbital & & & \\
& & & \\
& & & \\
\end{tabular}

Tabela adaptada de:D.K.; Dadhick A.P.Cytochrome P450 and drugs interactions. Indian. Jour. of. Pharm. 
De Menezes, F.G. De Mariz, L.C.V. Revista Eletrônica de Farmácia Vol. IX (1), 1 - 17, 2012.

Tabela3: Isoenzima CYP2C18 do Citocromo P-450 que é estimulada pela Fluoxetina ${ }^{(14)}$

\begin{tabular}{|l|c|c|l|l|}
\hline Isoenzima & \multicolumn{2}{|c|}{ Substrato } & $\begin{array}{l}\text { Medicamentos } \\
\text { inibidores }\end{array}$ & $\begin{array}{l}\text { Medicamentos } \\
\text { Indutores }\end{array}$ \\
\hline CYP2C18 & Ácido Retinóico & Lansoprazol & Cetoconazol & Fenitoina \\
& Diazepam & Naproxeno & Cimetidina & Rifampicina \\
& Diltiazem & Omeprazol & Fluoxetina & \\
& Fenitoína & Propranolol & & \\
& Imipramina & warfarina & & \\
\hline
\end{tabular}

Tabela adaptada de:D.K.; Dadhick A.P.Cytochrome P450 and drugs interactions. Indian. Jour. of. Pharm.

Tabela 5: Isoenzima CYP2D6 do Citocromo P-450 que é estimulada pela Fluoxetina(14)

\begin{tabular}{|l|l|l|l|l|}
\hline Isoenzima & \multicolumn{2}{|l|}{ Substrato } & \multicolumn{2}{|l|}{ Medicamentos inibidores } \\
\hline CYP2D6 & ADT & Imipramina & Amiodarona & Dimenidrinato \\
& Betabloqueadores & Mirtazapina & Amitriptilina & Haloperidol \\
& Captopril & Morfina & Bupropiona & Imipramina \\
& Carbamazepina & Paroxetina & Cimetidina & ISRS \\
& Carvediol & Prometazina & Citalopram & Metoclopramida \\
& Citalopram & Propranolol & Clomipramina & Propranolol \\
& Clorpromazina & Risperidona & Codeína & Ranitidina \\
& Codeína & Sertralina & Diltiazem & \\
& Clomipramina & Tramadol & & \\
& Clorfeniramina & Venlafaxina & & \\
& Fluoxetina & Zolpidem & & \\
& Haloperidol & & \\
\end{tabular}

Tabela adaptada de: D.K.; Dadhick A.P.Cytochrome P450 and drugs interactions. Indian. Jour. of. Pharm. 
De Menezes, F.G. De Mariz, L.C.V. Revista Eletrônica de Farmácia Vol. IX (1), 1 - 17, 2012.

Tabela 6: Isoenzima CYP3A4 do Citocromo P-450 que é estimulada pela Fluoxetina ${ }^{(14)}$

\begin{tabular}{|c|c|c|c|c|c|}
\hline Isoenzima & \multicolumn{3}{|l|}{ Substrato } & \multicolumn{2}{|c|}{ Medicamentos inibidores } \\
\hline CYP3A4 & $\begin{array}{l}\text { ADT } \\
\text { Albendazol } \\
\text { Alprazolam } \\
\text { Amiodarona } \\
\text { Anlodipina } \\
\text { Androgênios } \\
\text { Anticoagulantes } \\
\text { Benzodiazepínicos } \\
\text { Bloqueadores dos canais } \\
\text { de Ca²+ } \\
\text { Budesonida } \\
\text { Bupropiona } \\
\text { Carbamazepina } \\
\text { Cetoconazol } \\
\text { Citalopram } \\
\text { Claritromicina } \\
\text { Clindamicina } \\
\text { Clomipramina } \\
\text { Clonazepam } \\
\text { Clopidogrel } \\
\text { Clorpromazina } \\
\text { Codeína } \\
\text { Corticosteroide }\end{array}$ & $\begin{array}{l}\text { Teofilina } \\
\text { Varfarina } \\
\text { Venlafaxina } \\
\text { Diazepam } \\
\text { Diclofenaco } \\
\text { Digoxina } \\
\text { Diltiazem } \\
\text { Domperidona } \\
\text { Eritromicina } \\
\text { Escitalopram } \\
\text { Escopolamina } \\
\text { Estatinas } \\
\text { Etinilestradiol } \\
\text { Estrógeno } \\
\text { Finasterida } \\
\text { Fluoxetina } \\
\text { Haloperidol } \\
\text { Hidrocortisona } \\
\text { Hipoglicemiantes } \\
\text { Inibidores da HMG- } \\
\text { CoA }\end{array}$ & $\begin{array}{l}\text { Inibidores de protease } \\
\text { venlafaxina } \\
\text { Dexametasona } \\
\text { Montelucast } \\
\text { Nifedipina } \\
\text { Omeprazol } \\
\text { Itraconazol } \\
\text { Lansoprazol } \\
\text { Levonorgestrel } \\
\text { Loratadina } \\
\text { Lorazepam } \\
\text { Losartano } \\
\text { prednisolona } \\
\text { Miconazol } \\
\text { Pantoprazol } \\
\text { Paracetamol } \\
\text { Progestágenos } \\
\text { Prometazina } \\
\text { Sertralina } \\
\text { Sibutramina } \\
\text { Sildenafil }\end{array}$ & $\begin{array}{l}\text { Ácido valpróico } \\
\text { Amiodarona } \\
\text { Antifúngicosimidaz } \\
\text { ólicos } \\
\text { Atorvastatina } \\
\text { Azitromicina } \\
\text { Cetoconazol } \\
\text { Cimetidina } \\
\text { Diltiazem } \\
\text { Eritromicina } \\
\text { Etinilestradiol } \\
\text { Fluconazol } \\
\text { Fluoxetina } \\
\text { Gestodeno } \\
\text { Imipramina } \\
\text { Inibidores da } \\
\text { HMG-CoA } \\
\text { Inibidores da } \\
\text { protease }\end{array}$ & $\begin{array}{l}\text { ISRS } \\
\text { Inibidores da } \\
\text { transcriptase reversa } \\
\text { Itraconazol } \\
\text { Macrolídeos } \\
\text { Metronidazol } \\
\text { Miconazol } \\
\text { Midazolam } \\
\text { Nifedipina } \\
\text { Omeprazol } \\
\text { Progesterona } \\
\text { Propranolol } \\
\text { Quinolonas } \\
\text { Ranitidina } \\
\text { Sildenafil } \\
\text { Sinvastatina } \\
\text { Testosterona } \\
\text { Verapamil }\end{array}$ \\
\hline
\end{tabular}

Tabela adaptada de: D.K.; Dadhick A.P.Cytochrome P450 and drugs interactions. Indian. Jour. of. Pharm.

\section{MATERIAIS E MÉTODOS}

Estudo de utilização de medicamentos, prospectivo, com questionário estruturado, questões abertas e fechadas, aplicadas a pacientes atendidos na Unidade Básica de Saúde do Parque Novo Mundo II, localizada na Zona Norte do Município de São Paulo. Foram entrevistados pacientes do SUS, que apresentam prescrição contendo o medicamento fluoxetina. O questionário foi aplicado após o consentimento do paciente, assinatura de termo de consentimento livre e esclarecido. As principais informações que foram obtidas através do questionário são: dados demográficos (idade, sexo, escolaridade profissão) medicamentos em uso, hábitos, efeitos adversos percebidos pelo paciente, interações medicamentosas (consulta ao "Drug Interaction Checker", Medscape: 
De Menezes, F.G. De Mariz, L.C.V. Revista Eletrônica de Farmácia Vol. IX (1), 1 - 17, 2012.

www.medscape.com). Os dados foram coletados e armazenados em programa

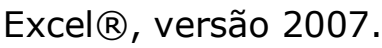

\section{RESULTADOS}

De um total de 44 pacientes entrevistados 37 pacientes $(84,40 \%)$ foram mulheres e 7 pacientes $(15,60 \%)$ homens. A faixa etária foi variada sendo entrevistados, $5(11,11 \%)$ com idade de 18 à 24 anos, $4(8,89 \%)$ com idade de 25 à 30 anos, $5(11,11 \%)$ com idade de 31 à 35 anos, $8(17,78 \%)$ com idade de 41 à 45 anos, $6(13,33 \%)$ com idade de 46 à 50 anos, $6(13,33 \%)$ com idade de 51 à 55 anos, $1(4,44 \%)$ com idade de 56 à 60 anos, $4(8,89 \%)$ com idade de 61 à 65 anos e $5(11,11 \%)$ com idade de 66 à 70 anos.

Em relação ao nível de escolaridade dos pacientes entrevistados 4 pacientes $(8,89 \%)$ não eram alfabetizados, 13 pacientes $(28,89 \%)$ possuem 0 ensino fundamental incompleto, 6 pacientes $(13,34 \%)$ possuem o ensino fundamental completo, 1 paciente $(2,22 \%)$ possue $o$ ensino médio incompleto, 12 pacientes $(26,67 \%)$ possuem o ensino médio completo, 3 pacientes $(6,67 \%)$ possuem o ensino superior incompleto e 5 pacientes $(13,33 \%)$ possuem o ensino superior completo.

Quanto ao hábito de fumar do total de 44 pacientes, $38(86,67 \%)$ não tem esse hábito e $6(13,33 \%)$ sim. Sendo que 1 paciente $(16,67 \%)$ fuma ocasionalmente e $5(83,33 \%)$ fumam diariamente. Dos pacientes fumantes $2(33,33 \%)$ iniciaram o uso do cigarro com a faixa etária de 10 à 14 anos, 2 ( 33,33\%) com a faixa etária de 15 à 19 anos e $2(33,33 \%)$ a 'partir de 24 anos em diante.

Em relação ao uso de bebidas alcoólicas, 34 pacientes $(77,78 \%)$ não tinham esse hábito e 10 pacientes $(22,22 \%)$ fazem uso de bebidas alcoólicas. Dos pacientes que responderam positivamente $8 \quad(80,00 \%)$ bebem ocasionalmente, $2(20,00 \%)$ bebem uma vez por semana. Sendo que $2(20,00 \%)$ iniciaram o uso com a faixa etária de 10 à 14 anos, $4(40,00 \%)$ com a faixa etária de 15 à 19 anos, $1(10,00 \%)$ com a faixa etária de 20 à 24 anos, 1 (10,00\%) começaram com 24 anos em diante e 2 $(20,00 \%)$ não lembram a idade que iniciaram o uso de bebidas alcoólicas.

Quanto ao uso do medicamento fluoxetina 25 pacientes $(57,78 \%)$ nunca interromperam o uso e 19 (42,22\%)sim. Dos que responderam positivamente a interrupção $1(5,27 \%)$ não lembra o período de tempo, 7 (36,83\%) ficaram se a medicação de 1 à 15 dias, 1(5,26\%) de 15 à 30 dias, 1 (5,27\%) por aproximadamente de 30 dias, 3 (15,79\%) por aproximadamente 60 dias, 2 (10,53\%) por aproximadamente 90 dias, $1(5,27 \%)$ por aproximadamente 120 dias, 1 (5,27\% 
De Menezes, F.G. De Mariz, L.C.V. Revista Eletrônica de Farmácia Vol. IX (1), 1 - 17, 2012.

) por aproximadamente 180 dias, 1 $(5,27 \%)$ por aproximadamente 270 dias, 1 ( 5,27\%) por aproximadamente 2 anos.

Do total de pacientes 7 pacientes $(15,09 \%)$ não segue as orientações médicas, e $10(22,72 \%)$ informa ao médico todas substâncias e medicamentos que faz uso.

Quanto ao aparecimento dos efeitos adversos 11 (25\%) pacientes relataram ser assintomáticos sendo que 8 paciente haviam iniciado a uso da medicação a menos de 1 mês, e os outros usavam a medicação a 6 anos, 4 anos e 2 meses. Porém a grande maioria 33 pacientes (75\%) apresentaram sintomas indesejáveis, descritos na figura abaixo:

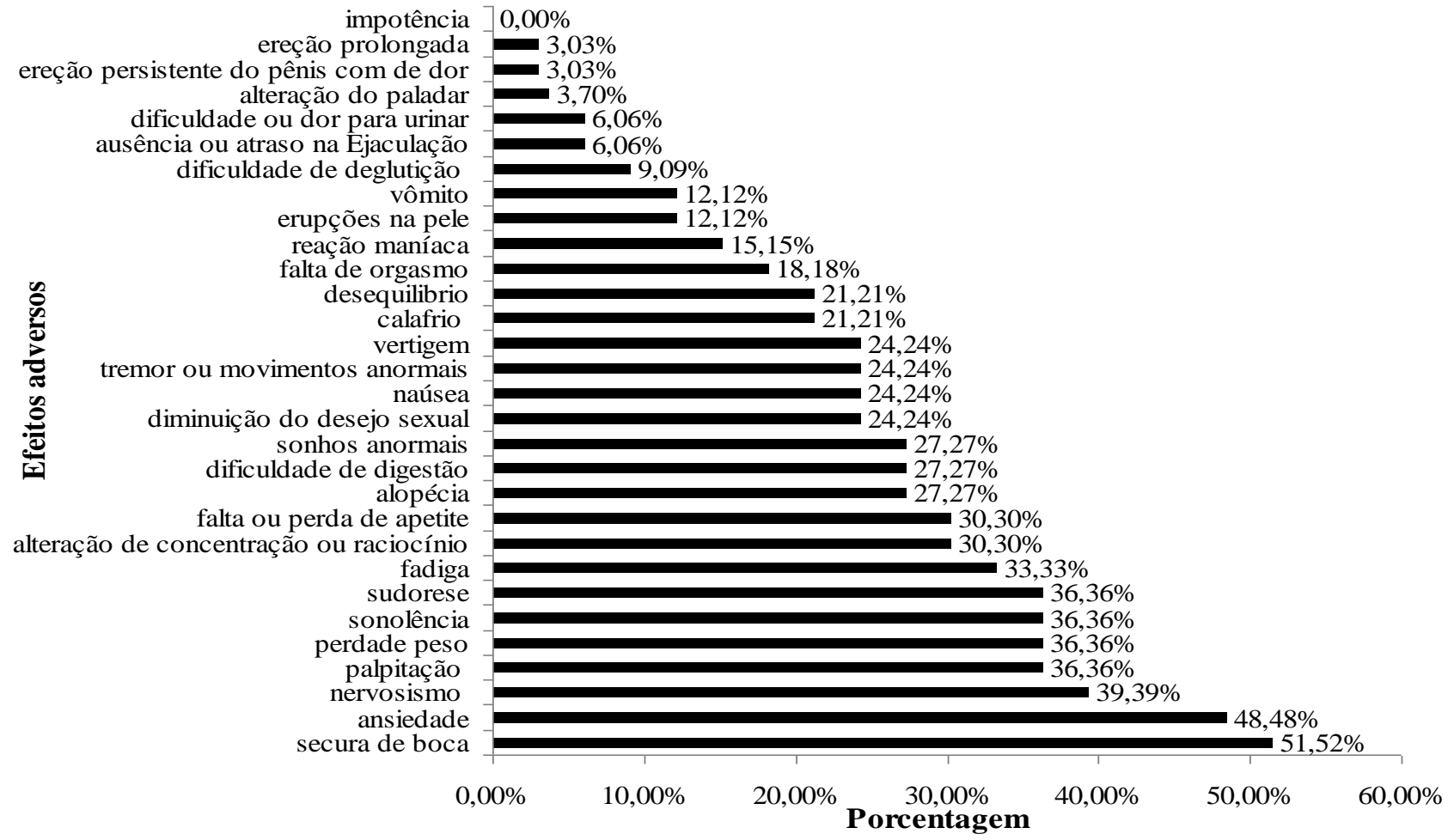

Figura 1: Principais efeitos adversos e suas frequencias nos pacientes entrevistados que utilizam fluoxetina, atendidos em UBS Parque Novo Mundo, Zona Norte, São Paulo.

Outro dado relevante os medicamentos utilizados concomitantemente a fluoxetina, foram encontradas as seguintes medicações : 
De Menezes, F.G. De Mariz, L.C.V. Revista Eletrônica de Farmácia Vol. IX (1), 1 - 17, 2012.

Tabela 7: Medicamentos utilizados em associação com a fluoxetina, dos pacientes entrevistados:

\begin{tabular}{|c|c|}
\hline Medicamentos que atuam no SNC & Código ATC \\
\hline Clonazepam, Diazepam & N03AE01 N05BA01 \\
\hline Sibutramina & A08AA10 \\
\hline Zolpidem & N05CF02 \\
\hline Haloperidol & N05AD01 \\
\hline Amitripitilina & N06AA09 \\
\hline Paracetamol e combinações & N02BE51 \\
\hline Topiramato & N03AX11 \\
\hline \multicolumn{2}{|l|}{ Medicamentos Anti-hipertensivos } \\
\hline Nifedipino. anlodipino & C08CA05, C08CA01 \\
\hline Captopril , enalapril, lisinopril & C09AA01, C09AA02, C09AA03 \\
\hline Hidroclorotiazida & C03AA03 \\
\hline Atenolol & C07AB03 \\
\hline Hidroclorotiazida e combinações & C03AX01 \\
\hline Verapamil & C08DA01 \\
\hline Propitilnitrato & C01DA07 \\
\hline Valsartano + amlodipino & C09DB01 \\
\hline \multicolumn{2}{|l|}{ Medicamentos Hipoglicemiantes } \\
\hline Metformina & A10BA02 \\
\hline Glibenclamida & A10BB01 \\
\hline \multicolumn{2}{|l|}{ Medicamentos anti-ulcerosos } \\
\hline Cimetidina & A02BA01 \\
\hline Omeprazol, pantoprazol & $\mathrm{A} 02 \mathrm{BC} 05, \mathrm{~A} 02 \mathrm{BC} 02$ \\
\hline \multicolumn{2}{|l|}{ Medicamentos para o sistema endócrino } \\
\hline $\begin{array}{l}\text { Gestodeno + Etinilestradiol, norethisterone + } \\
\text { Etinilestradiol, drospirenona + etinilestradiol, } \\
\text { levonorgestrel +etinilestradiol }\end{array}$ & G03AA10, G03AA05, G03AA07, G03AA12 \\
\hline Ciproterona + etinilestradiol & G03HB01 \\
\hline Levotiroxina sódica & H03AA01 \\
\hline \multicolumn{2}{|l|}{ Antibióticos } \\
\hline Cefalexina & J01DB01 \\
\hline Amoxicillina & J01CA04 \\
\hline \multicolumn{2}{|c|}{ Antiinflamatórios, antipiréticos e antiespasmódico } \\
\hline Dipirona & N02BB02 \\
\hline Ibuprofeno & C01EB16, G02CC01, M01AE01, M02AA13 \\
\hline Ácido Acetilsalicilico & A01AD05, B01AC06, N02BA01 \\
\hline Diclofenaco & D11AX18, M01AB05, M02AA15, S01BC03 \\
\hline
\end{tabular}


De Menezes, F.G. De Mariz, L.C.V. Revista Eletrônica de Farmácia Vol. IX (1), 1 - 17, 2012.

\begin{tabular}{|c|c|}
\hline Escopolamine & A04AD01, N05CM05, S01FA02 \\
\hline Nimesulida & M01AX17 \\
\hline Naproxeno & G02CC02, M01AE02, M02AA12 \\
\hline Ácido mefenamico & M01AG01 \\
\hline \multicolumn{2}{|l|}{ Corticóides } \\
\hline Beclometasona & A07EA07, D07AC15, R01AD01 R03BA01 \\
\hline Prednisone & A07EA03, \\
\hline \multicolumn{2}{|c|}{ Inibidores enzimáticos } \\
\hline Anastrozol & L02BG03 \\
\hline \multicolumn{2}{|l|}{ Antiasmáticos } \\
\hline Fenoterol & G02CA03, R03AC04, R03CC04 \\
\hline Ipratrópio & R01AX03, R03BB01 \\
\hline
\end{tabular}

Fonte para elaboração da tabela 7: WHO Collaborating Centre for Drud Statistics Methodology, ATC/DDD Index 2010.

Algumas possíveis interações medicamentosas que ocorre com o uso de fluoxetina e outras substâncias, veremos na tabela 8.

Tabela 8: Possíveis interações medicamentosas com o uso de fluoxetina.

\begin{tabular}{|l|l|}
\hline Principais interações medicamentosas com o uso da fluoxetina/causa \\
\hline Benzodiazepínicos & $\begin{array}{l}\text { Aumento dos níveis plasmáticos dos } \\
\text { benzodiazepínicos. }\end{array}$ \\
\hline Clozapina & Aumento dos níveis plasmáticos da clozapina. \\
\hline Warfarina & Aumento dos níveis plasmáticos da warfarina. \\
\hline Antidepressivos Tricíclicos & $\begin{array}{l}\text { Potencialização dos efeitos dos antidepressivos } \\
\text { tricíclicos. }\end{array}$ \\
\hline Neurolépticos & Aumento dos níveis plasmáticos dos neurolépticos. \\
\hline
\end{tabular}

\section{DISCUSSÃO}

O texto traz uma reflexão sobre o uso do medicamento fluoxetina. Esse ISRS opção clássica para o tratamento das depressões, distúrbios alimentares (bulimia nervosa), perturbação obsessivocompulsiva e síndrome do pânico, porém pode levar a efeitos adversos indesejados ${ }^{(9)}$. "A Organização Mundial de Saúde (OMS) define saúde não apenas como a ausência de doença, mas como a situação de perfeito bem-estar físico, mental e social'(16). Considerando essa definição deve se avaliar se o uso realmente esta restabelecendo á saúde do paciente. Pode-se ainda verificar algumas associações medicamentosas prejudiciais a saúde do paciente, sendo quase sempre acompanhadas da não informação ao clínico de várias prescrições utilizadas. Dos medicamentos utilizados comitantemente com a fluoxetina merece atenção maior os inibidores do CYP 2C9 : cimetidina, 
De Menezes, F.G. De Mariz, L.C.V. Revista Eletrônica de Farmácia Vol. IX (1), 1 - 17, 2012.

diazepam, diclofenaco, omeprazol, lembrando que o próprio uso da fluoxetina inibe esse citocromo. A cimetidina e a fluoxetina também são inibidores do CYP2C18. Os medicamentos: amitriptilina, cimetidina, haloperidol e a fluoxetina são inibidores do CYP2D6. Os fármacos cimetidina, omeprazol, verapamil são inibidores do CYP3A4 ${ }^{(12)}$. Merece ainda uma atenção especial a associação anorexígena de fluoxetina e sibutramina, onde ocorre a somatória de dois mecanismos de ação parecidos, a inibição seletiva da recaptação de serotonina e a inibição de da recaptação de serotonina respectivamente. Podendo levar a uma síndrome serotonérgica caracterizada por alterações cognitivas e comportamentais (confusão, hipomania, agitação), do sistema nervoso autônomo (diarréia, febre, diaforese, efeitos na pressão arterial, náuseas e vômitos) e neuro-musculares (mioclonias, hiperreflexia, incoordenação e tremores $)^{(9,11)}$.

É relevante notar que, na imensa maioria das prescrições, a fluoxetina foi indicada para mulheres. Esses valores também são observados em várias regiões do mundo. Com uma média de 2 mulheres para cada homem ${ }^{(17,18)}$. Fato este pode ser explicado pela maior prevalência de casos de transtornos depressivos e ansiedade ocorrer em pacientes do sexo feminino, enquanto que o sexo masculino apresenta maior prevalência de abuso de substâncias psicoativas, como o álcool, além de transtornos esquizofrênicos, compulsão, déficit de atenção e hiperatividade na infância e vida adulta ${ }^{(17)}$.

Os hormônios sexuais femininos, principalmente o estrógeno, agem na modulação do humor, o que, parcialmente, explicaria a maior parte dos transtornos do humor e de ansiedade na mulher. As variações hormonais sexuais poderiam influenciar a modulação do sistema neuroendócrino feminino. Seria uma hipótese de susceptibilidade biológica para explicar a diferença nas prevalências de transtornos de humor entre homens e mulheres. Outra possibilidade seria o desequilíbrio na interação entre eixo hipotálamo-hipófise-gônada e demais neuromoduladores, sendo que o ritmo reprodutivo da mulher pode apresentar suscetibilidade a fatores psicossociais, ambientais e fisiológicos ${ }^{(17)}$.

Em relação ao tratamento de TOC estima-se que ocorra uma maior prevalência em adultos do sexo feminino $^{(19)}$. Os transtornos alimentares aparecem em sua maioria de 90 à $95 \%$ em mulheres, e um aumento do número de casos tem sido observado em homens e mulheres de todas as idades ${ }^{(20)}$.

Os transtornos alimentares que tem como indicação de tratamento o uso da fluoxetina são mais prevalentes em 
De Menezes, F.G. De Mariz, L.C.V. Revista Eletrônica de Farmácia Vol. IX (1), 1 - 17, 2012.

adolescentes e adultos jovens pertencentes a todos os grupos étnicos, sendo aproximadamente dez vezes mais comuns em mulheres que em homens, também os transtornos de ansiedade são mais frequentes no sexo feminino ${ }^{(19,20)}$.

Em relação à prevalência em diferentes faixas etárias dos pacientes os transtornos depressivos constituem um grupo de patologias com alta e crescente prevalência na população em geral. Diz a Organização Mundial de Saúde, que nas próximas duas décadas haverá necessidades de saúde da população mundial em relação ao tratamento de transtornos depressivos. Porém percebese que a maioria dos transtornos depressivos tem início entre 20 e 40 anos, de forma similar ao sexo, também não há porque pensar que a idade seja um fator de risco isoladamente e sim associado a fatores sociais, no caso dos jovens há maior risco ${ }^{(21)}$. Da mesma forma merece uma atenção especial a depressão em idosos. No Brasil as taxas de prevalência variam entre $5 \%$ e $35 \%$, quando consideramos as diferentes formas e a gravidade da depressão(22).

Pessoas com baixa escolaridade e economicamente desfavorecidas possuem maiores risco de apresentar depressão, fatores sócio-econômicos podem modificar o efeito de estressores sobre as pessoas apoio social e econômico são fatores protetores que o meio é capaz de fornecer aos indivíduos possibilitando-os a lidar com fatores estressores ${ }^{(23)}$.

\section{CONCLUSÃO}

Observamos várias associações medicamentosas que poderiam causar dano ao paciente, a saber: fluoxetina com sibutramina, amitriptilina, omeprazol, haloperidol, entre outros. Tais interações poderiam causar as seguintes situações, por exemplo: aumento dos níveis plasmáticos do omeprazol pela inibição enzimática causada pela fluoxetina; potencializar o efeito da recaptura de serotonina, causando síndrome serotonérgica, no caso da amitriptilina e sibutramina; potencialização do efeito da fluoxetina pelo haloperidol que aumenta seus níveis plasmáticos e os dois fármacos associados podem causar prolongamento do intervalo QT.

Baixa porcentagem dos pacientes informou ao médico todas as substâncias que utiliza, tal atitude aumenta a possibilidade de interações medicamentosas e predisposição a efeitos adversos. Portanto, o estudo ressalta a importância da necessidade de obtenção detalhada sobre o uso de medicamentos: na anamnese, a participação ativa do paciente enquanto informante consciente do uso de medicamentos durante a consulta médica; o acompanhamento 
De Menezes, F.G. De Mariz, L.C.V. Revista Eletrônica de Farmácia Vol. IX (1), 1 - 17, 2012.

farmacoterapêutico realizado pelo farmacêutico durante a dispensação e o questionamento dos demais profissionais da saúde quanto ao uso de medicamentos; reforçando o papel da equipe multidisciplinar na cadeia do medicamento. A não comunicação do paciente é fator importante que pode estar ligada à baixa escolaridade da maioria dos entrevistados. Esse fator deve ser Também se faz necessária a reavaliação da necessidade prescrição da fluoxetina, preconizando sempre o uso racional da mesma.

\section{AGRADECIMENTOS}

Agradecemos a farmacêutica Marcela Reis por participar da etapa de coleta dos dados.

avaliado tanto pelo médico durante a consulta quanto pelo farmacêutico no ato da dispensação do medicamento.

\section{REFERÊNCIAS BIBLIOGRÁFICAS}

1. Chiodi MB, Marziale MHP. Riscos ocupacionais para trabalhadores de Unidades Básicas de Saúde: revisão bibliográfica. Acta paul. Enferm. 2006;19(2):212-7.

2. Silvia AM, Bussinger ECA. O poder judiciário e o fornecimento de medicamentos no SUS: uma análise da macroregião sulsul do Espírito Santo. Infarma. [Internet]. 2010 [cited 2012 fev 04];22(11/12):34-44. Aviable from: http:www.cff.org.br/sistemas/geral/revista/pdf/129/069a132_infarma_pb79.pdf.

3. Pissato S, Prado JN, Morais EC, Foppa T, Murakami FS, Silva MAS. Avaliação da Qualidade de Cápsulas de Cloridrato de Fluoxetina. Acta Farm. Bonaerense. $2006 ; 25(4): 550-4$.

4. Correia DT, Guerreiro DF, Oliveira S, Figueira ML. Diferenças farmacodinâmicas e farmacocineticas entre os SSRI. Acta Med Port. 2007;20:167-174.

5. Wong DT, Bymaster FP, Engleman EA. Prozac (fluoxetine, Lilly 110140), the first selective serotonin uptake inhibitor and an antidepressant drug: twenty years since its first publication. Life Sci. 1995;57(5):411-41. 
De Menezes, F.G. De Mariz, L.C.V. Revista Eletrônica de Farmácia Vol. IX (1), 1 - 17, 2012.

6. Top 200 Generic Drugs by Units in 2006. [Internet]. [cited 2009 jun 14]. Aviable from: http://drugtopics.modenmedicine.com/drugtopics/data/articlestandart/ drugtopics/09 2007/407652/article.pdf.

7. Mancini MC, Halpern A. Aspectos fisiológicos do balanço energético. Arq Bras Endocrinol Metab. 2002;46(3):231-48.

8. Banki CM. Serotonin: La chica de moda in Biological Psychiatry?. Biol Psychiatry. 1991;29(29):949-52.

9. Carlini EA, Noto AR, Nappo SA, Sanchez ZVDM, Fanco VLS, Silva LCF, et al Fluoxetina: indícios de uso inadequado. J bras psiquiatr. 2009;58(2):97-100.

10. BRASIL. Agência Nacional de Vigilância Sanitária. Resolução - RDC no. 140, de 29 de maio de 2003. Republicada no D.O.U de 24/09/2003.

11. Moreno RA, Moreno DH, Soares MBM. Psicofarmacologia de antidepressivo. Rev Bras Psiquiatr. 1999;(21):24-40.

12. Audi EA; Pussi FD. Isoenzimas do CYP450 e biotransformação de drogas. Acta Sci. $2000 ; 22(2): 599-604$.

13. Marcolin MA; Cantarelli MG; Junior MG. Interações farmacológicas entre medicações clínicas e psiquiátricas. Rev. Psiq. Clín. 2004;31(2):70-81.

14. Badyal DK, Dadhich AP. Cytochrome P450 and drug interactions. Indian J Pharmcol. 2002;(34):289-91.

15. Cordeiro CHG, Chung MC, Do Sacramenteo LVS. Interações medicamentosas de fitoterápicos e fármacos: Hypericumperforatum e Piper methysticum. Rev. Bras. Farmacogn. 2005;15(3):272-78.

16. Segre M, Ferraz FC. O conceito de saúde. Rev. Saúde Pública. $1997 ; 31(5): 538-42$. 
De Menezes, F.G. De Mariz, L.C.V. Revista Eletrônica de Farmácia Vol. IX (1), 1 - 17, 2012.

17. Andrade LHSG, Viana MC, Silveira CM. Epidemiologia dos transtornos psiquiátricos na mulher. Rev. Psiq. Clín. 2006;33(2);43-54.

18. Almeida FN, Lessa I, Magalhães L, Araújo MJ, Aquino E, James AS, et al. Social inequality and depressive disorders in Bahia, Brazil: interactions of gender, ethnicity, and social class. Soc Sci Med. 2004;59(7):1339-53.

19. Torres AR, Lima MCP. Epidemiologia do transtorno obsessivo-compulsivo: uma revisão. Rev Bras Psiquiatr. 2005;27(3):237-42.

20. Lima MS. Epidemiologia e impacto social. Rev Bras Psiquiatr. 1999;21(1):0105.

21. Leite VMM, Carvalho EMF, Barreto KML, Falcão IV. Depressão e envelhecimento: estudo nos participantes do Programa Universidade Aberta à Terceira Idade. Rev. Bras. Saude Matern. Infant. 2006;6(1) 31-8.

22. Martin D, Quirino J, Mari J. Depressão entre mulheres da periferia de São Paulo. Rev. Saúde Pública. 2007;41(4):591-7.

23. Vilela JEM, Lamounier JA, Filho MAD, Neto JRB, Horta GM. Transtornos alimentares em escolares. children. J Pediatr. 2004;80(1):49-54. 